\title{
Assessment of Antibiotic Prescription in Respiratory Tract Infection at Hera General Hospital in Makkah \\ Almukarramah, KSA
}

\author{
Rafea B. ${ }^{1}$, Alharbi F. ${ }^{1}$, Alhazmi G. ${ }^{1}$, Alahmadi L. ${ }^{1}$, Almotiri M. ${ }^{1}$, Alharthi M. ${ }^{1}$, Alsulami M. ${ }^{1}$, Alamri R. ${ }^{1}$, \\ Alkhallaf R. ${ }^{1}$, Hettah R. ${ }^{2}$, Alhindi Y. ${ }^{3}$ \\ ${ }^{1}$ Pharm D interns students, Faculty of pharmacy, University of Umm Al-Qura, Makkah, KSA \\ ${ }^{2}$ Drug information center, Internal Pharmacy, Hera General hospital, Makkah, KSA \\ ${ }^{3}$ Assistant Professor in clinical pharmacy, Faculty of pharmacy, University of Umm Al-Qura, Makkah, KSA
}

*Corresponding Author - Alhindi Y.; yzhindi@uqu.edu.sa

Received 11 March 2019;

Accepted 25 March 2019;

Published 28 March 2019;

\begin{abstract}
Introduction: In general practice, approximately two-thirds of antibiotic prescriptions are for respiratory tract infections. Acute respiratory tract infections, which include acute uncomplicated bronchitis, pharyngitis, rhinosinusitis and the common cold, are often common reasons for acute outpatient visits to physicians and antibiotic prescriptions for adults. The improper treatment of acute respiratory tract infections is now an urgent public health threat, as it is an important contributor to antibiotic resistance. In developing countries, including Saudi Arabia, antibiotics are prescribed excessively and are often obtained without prescription. Aim: The aim of this study was to gather information on current antibiotic prescribing rates for respiratory tract infection in Hera General Hospital Makkah Almukarramah, Saudi Arabia. Method: A crosssectional study, incorporating systematic sampling on clinical data from antibiotic prescriptions in hospitalised and emergency departments at Hera General Hospital, was conducted. From September 2017 to April 2018, approximately 500 prescriptions were collected from each hospitalised and emergency pharmacy for patients with respiratory tract infections. Results: The most prescribed antibiotics for respiratory tract infections came from the penicillin group and ciprofloxacin. The prescribing of antibiotics for respiratory tract infections was more prevalent amongst males than females. Potential over-prescribing of antibiotics for upper respiratory tract infections specially patients with otitis media especially in children without proper lab investigations.
\end{abstract}

\section{Introduction}

Antibiotics are amongst the most commonly prescribed drugs in hospital settings. No other class of drug can compete with antibiotic agents in terms of potential disease cure and the extent to which mortality has been reduced over the last 70 years. Because of increasing health care costs, a lack of uniformity in drug prescribing and the emergence of antibiotic resistance, the monitoring and control of antibiotics is a growing concern. Similarly, to combat these issues, strict antibiotic policies and prescribing guidelines are needed ${ }^{[1]}$.

Essential medicines, as defined by the World Health Organisation (WHO) are "those drugs that satisfy the health care needs of the majority of the population; they should therefore be available at all times in adequate amounts and in appropriate dosage forms, at a price the community can afford" ${ }^{[2]}$.
Approximately half of all medicines are prescribed, dispensed or sold inappropriately. Similarly, approximately half of all patients fail to use them correctly. The overuse, underuse or misuse of medicines results in the wastage of scarce resources and causes widespread health hazards, e.g. (1) the prescription of too many medicines per patient ("poly-pharmacy"), (2) the inappropriate use of antimicrobials, often in inadequate dosages for non-bacterial infections, (3) the overuse of injections when oral formulations are more appropriate, (4) the failure to prescribe in accordance with clinical guidelines, (5) inappropriate self-medication of prescription-only medicines and (6) non-adherence to dosing regimens ${ }^{[2]}$.

Antibiotic misuse is a worldwide problem in both developed and developing countries ${ }^{[3]}$. Antibiotics are grossly abused in these developing countries, e.g. in Africa, medicines are purchased without prescription in local pharmacies and drug stores and they are used/prescribed inappropriately thanks to over-zealous desires to treat every infection. The need to formulate and adopt stringent 
policies for appropriate antibiotic usage is now more pressing in developing nations, where the highest levels of misuse and abuse are encountered.

Well-established antibiotic policies go a long way towards achieving reductions in irrational prescribing. Moreover, the issue can be addressed with a reasonable degree of success when prescribing physicians have adequate medicinal knowledge, e.g. the properties of chemotherapeutic agents, the underlying disease pathogen, and their susceptibility or resistance profiles to commonly available antibiotics. Patient compliance is also vitally important and must be addressed ${ }^{[4]}$.

Since their discovery at the beginning of the 20th century, antimicrobial agents (antibiotics and related medicinal drugs) have reduced the threat of many serious infectious diseases. These drugs have contributed to significant life expectancy gains during the latter part of the last century. These gains are now seriously jeopardised by recent developments: the emergence and spread of microbes that are resistant to cheap and ineffective first-choice, or "first-line" drugs ${ }^{[2]}$.

Antimicrobial resistance (AMR) is a serious public health problem. In the last decade, antimicrobial resistance has increased worldwide ${ }^{[5]}$. Many microbes (bacteria, viruses, protozoa) that cause infectious disease no longer respond to common antimicrobial drugs (antibiotics, antivirals and antiprotozoal drugs). The problem is serious; the level of antibiotics consumed in a community is directly related to the antibiotic resistance found in the community ${ }^{[6]}$.

For instance, an ecological study linked penicillin-nonsusceptibility in Streptococcus pneumoniae with lactam and macrolide use in 12 European countries. Penicillin non-susceptible S. pneumoniae and macrolide-resistant S. pneumoniae are markers of resistance of antibiotics commonly used as first-line drugs for respiratory tract infections (RTIs). Although penicillin-resistant S. pyogenes has never been observed to date, the increasing rates of macrolide-resistant group A Streptococci pose considerable clinical problems in many countries ${ }^{[7]}$.

Inappropriate use of antibiotics, particularly for respiratory infections (RIs), has contributed significantly to antibiotic resistance $^{[8]}$. When an individual is infected with an antibioticresistant bacterium, not only is treatment more difficult, but the antibiotic-resistant bacterium may spread to other populations ${ }^{[9]}$. Equally, further concerns are demonstrated when new antibiotics are developed, there may be pressures to limit its use for the treatment of serious cases or when there is resistance to other antibiotics $^{[10]}$.

Respiratory tract infections (RTIs) are the most prevalent infectious diseases in many societies. They are a common reason for patients visiting emergency departments, as well as $20-40 \%$ of outpatient clinics and about $12-35 \%$ of inpatient attendance in a general hospital ${ }^{[2]}$. RTIs are defined as any infectious disease of the upper or lower respiratory tract. Upper respiratory tract infections (URTIs) include the common cold, laryngitis, pharyngitis/tonsillitis, acute rhinitis, acute rhinosinusitis, and acute otitis media. These infections are usually benign, transitory and self-limited. Lower respiratory tract infections (LRTIs) include acute bronchitis, bronchiolitis, pneumonia and tracheitis. These infections, especially pneumonia, can be severe or fatal ${ }^{[11,12]}$.
The most common respiratory tract infections in Saudi Arabia are URTIs ${ }^{[13,14]}$. In 2000, a Saudi Arabian study analysed prescriptions for acute RTIs and found that approximately $65.8 \%$ accounted for URTIs ${ }^{[15]}$. RTIs are mainly caused by the interaction of viruses with bacteria and vice versa. Therefore, knowledge of clinical manifestations differentiating viral from bacterial infections can promote the appropriate management of respiratory infections, in consultation with definitive clinical guidelines ${ }^{[16]}$. Both viruses and bacteria are etiological factors participating in URTI development, however, most infections are caused by viruses. Importantly, the management of such infections require symptomatic treatments such as antihistamines, antipyretics, antiinflammatory agents, antitussive, expectorants and decongestants, but not antibiotics ${ }^{[17,18]}$.

The prescription of antibiotics is more frequent for URTI patients. Approximately $75 \%$ of adults diagnosed with URTIs are prescribed antibiotics ${ }^{[19,20,21]}$. There should be no role for antibiotics in the management of the common cold or URTIs. There are many problems associated with antibiotic overuse; (1) the development of antibacterial resistance, (2) increases in chronic disease complications, (3) increased drug and health services costs, and (4) the development of side effects. The use of antibiotics is considered the most important factor in the development of bacterial resistance to antimicrobial drugs ${ }^{[22]}$. Several studies have focused on the increased inappropriate consumption of antibiotics in Saudi Arabia ${ }^{[23,24]}$. Antibiotics were the second most used drug class in Saudi Arabia and represented approximately 17\% of the most frequently used drugs in Saudi Arabia ${ }^{[25]}$.

Previous studies conducted in Saudi Arabia have shown that very high percentage of antibiotics were prescribed for acute RTIs, even though the majority of cases were not bacterial in nature ${ }^{[26,27]}$. According to a previous study of antibiotic misuse of URTIs in King Khalid University Hospital, College of Medicine, King Saud University, Riyadh, Saudi Arabia, $45.5 \%$ of participants used antibiotics without a prescription and $10.8 \%$ used the mover a pharmacist's advice. Also, $17.3 \%$ of participants forced physicians to prescribe antibiotics ${ }^{[28]}$. This study aims was to gather detailed information on current antibiotic prescribing rates for RTIs in Hera General Hospital in Makkah, Saudi Arabia.

\section{Methodology}

\section{Study design}

From September 2017 to April 2018, we carried out a crosssectional study, incorporating systematic sampling, on clinical data from antibiotic prescriptions in hospitalised and outpatients at Hera General Hospital.

\section{Study setting}

The study was conducted in both hospitalised and Emergency Room (ER).

\section{Sample size}

500 RTI patient prescriptions were collected from each hospitalised and ER pharmacy.

\section{Data collection}

Prescriptions were randomly examined to record information about prescribing indicators. The standard WHO prescribing indicators are calculated as:

- The mean number of antibiotic drugs/prescriptions, 
- The percentage of antibiotics prescribed from all prescribed drugs.

\section{Inclusion criteria}

Inpatients and Casualty patients (seen on an emergency basis, 24 hours a day, 7 days a week).

\section{Exclusion criteria}

Chronic clinic patients (e.g. diabetes and hypertension).

\section{Data analysis}

Descriptive statistics were used to analyse the data. Once all data were captured and data forms completed, antibiotic numbers, types of antibiotics and types of conditions were tabulated in an Excel spreadsheet. The results were extracted using Pivot charts and tables. t-tests were carried out with $\mathrm{P}$ scores $(\mathrm{P}<0.005)$.

\section{Ethics}

Ethical approval was obtained from the Hera General Hospital pharmacy director.

\section{Results}

\section{Demographic data from prescriptions:}

Inpatient population characteristics are shown in Table 1A. More than half of participants were male $(54 \%)$, whereas $46 \%$ were female. Age grouping showed that participants of 65 years of age or more were the highest attendees $(46 \%)$.

Table 1A: Demographics of inpatient participants

\begin{tabular}{|c|c|c|}
\hline \multirow{3}{*}{ Gender } & & Percentage \\
\cline { 2 - 3 } & Male & $54 \%$ \\
\cline { 2 - 3 } & Female & $46 \%$ \\
\cline { 2 - 3 } A to 13 years & $10 \%$ \\
\cline { 2 - 3 } & $16-24$ & $3 \%$ \\
\cline { 2 - 3 } & $25-34$ & $5 \%$ \\
\cline { 2 - 3 } & $35-44$ & $7 \%$ \\
\cline { 2 - 3 } & $45-54$ & $13 \%$ \\
\cline { 2 - 3 } & $55-64$ & $16 \%$ \\
\cline { 2 - 3 } & $65+$ & $46 \%$ \\
\hline
\end{tabular}

The characteristics of the ER population are shown in Table1B. The age of included subjects ranged from infancy to 13 years old in $60 \%, 16-24$ years in 20\%, 25-34 years in 5\%, 34-44 years in 1\%, $45-54$ years in $1 \%, 55-64$ in 3\% and $10 \%$ aged more than 65 years old. More than half of participants were male (51\%), whereas $49 \%$ were female. Age grouping showed that participants aged between infancy $(0)$ and 13 years old were the highest attendees $(60 \%)$.

Table.1 B: Demographic characteristics of ER participants

\begin{tabular}{|c|c|c|}
\cline { 2 - 3 } Gender & Male & Percentage \\
\cline { 2 - 3 } & Female & $49 \%$ \\
\cline { 2 - 3 } Age (yrs) & $\begin{array}{c}\text { O to 13 } \\
\text { years }\end{array}$ & $60 \%$ \\
\cline { 2 - 3 } & $16-24$ & $20 \%$ \\
\cline { 2 - 3 } & $25-34$ & $5 \%$ \\
\cline { 2 - 3 } & $35-44$ & $1 \%$ \\
\cline { 2 - 3 } & $45-54$ & $1 \%$ \\
\cline { 2 - 3 } & $55-64$ & $3 \%$ \\
\cline { 2 - 3 } & $65+$ & $10 \%$ \\
\hline
\end{tabular}

\section{Conditions for which antibiotics were prescribed:}

For inpatients, the most common URTI treated with antibiotics was pneumonia $(33.8 \%)$. Next came aspiration pneumonia (30.3\%), followed by bronchopneumonia (26.9\%). The least common RTIs treated with antibiotics were bronchial asthma, acute pulmonary edema, bronchiectasis and hospital acquired pneumonia $(0.6 \%$ each) (Table 2A).

On the other hand, the most common infection treated in the ER were URTIs, accounting for $71 \%$. After this, tonsillitis accounted for $10.7 \%$ followed by asthma (5.8\%) and otitis media (3.2\%). The least common infection was bronchitis $(0.6 \%)$ (Table $2 \mathrm{~B})$.

In certain cases, prescriptions covered multiple diagnoses other than respiratory infection. However, the duration of prescriptions ranged from one to seven days.

Table 2A: Most common URTIs in inpatients at Hera General Hospital Medical Ward

\begin{tabular}{|c|c|}
\hline Disease & Percentage \\
\hline Pneumonia & $33.8 \%$ \\
Aspiration pneumonia & $30.3 \%$ \\
Bronchopneumonia & $26.9 \%$ \\
Bronchitis & $3.5 \%$ \\
Community-acquired pneumonia & $2.5 \%$ \\
Interstitial lung disease & $1.0 \%$ \\
Bronchial asthma & $0.5 \%$ \\
Acute pulmonary edema & $0.5 \%$ \\
Bronchiectasis & $0.5 \%$ \\
Hospital acquired pneumonia & $0.5 \%$ \\
\hline
\end{tabular}

Table 2B: Most common URTIs in ER at Hera General Hospital

\begin{tabular}{|c|c|}
\hline Disease & Percentage \\
\hline Tonsillitis & $55.0 \%$ \\
Bronchial asthma & $22.5 \%$ \\
Bronchitis & $4.0 \%$ \\
Otitis media & $3.2 \%$ \\
Acute Pharyngitis & $3.0 \%$ \\
Acute Tonsillitis & $2.0 \%$ \\
Bronchial asthma/bronchopneumonia & $2.0 \%$ \\
Bronchitis / Bronchopneumonia & $2.0 \%$ \\
URTI with conjunctivitis & $2.0 \%$ \\
Bronchitis & $2.0 \%$ \\
\hline
\end{tabular}

Number and type of antibiotics prescribed per patient:

There were $471(58.9 \%)$ single antibiotic prescriptions, $72(13.1 \%)$ double antibiotic prescriptions, and $5(0.9 \%)$ triple antibiotic prescriptions.

A total of 22 different antibiotics, or in different combinations, were prescribed, totalling 201 occurrences for inpatient prescriptions. These comprised amoxicillin, augmentin, azithromycin, cefuroxime, cephalexin, ciprofloxacin and erythromycin was the most commonly prescribed antibiotic per patient either as a single agent or in combination with other antibiotics. Ciprofloxacin was the highest prescribed antibiotic $(30 \%)$ (Fig 1A) 


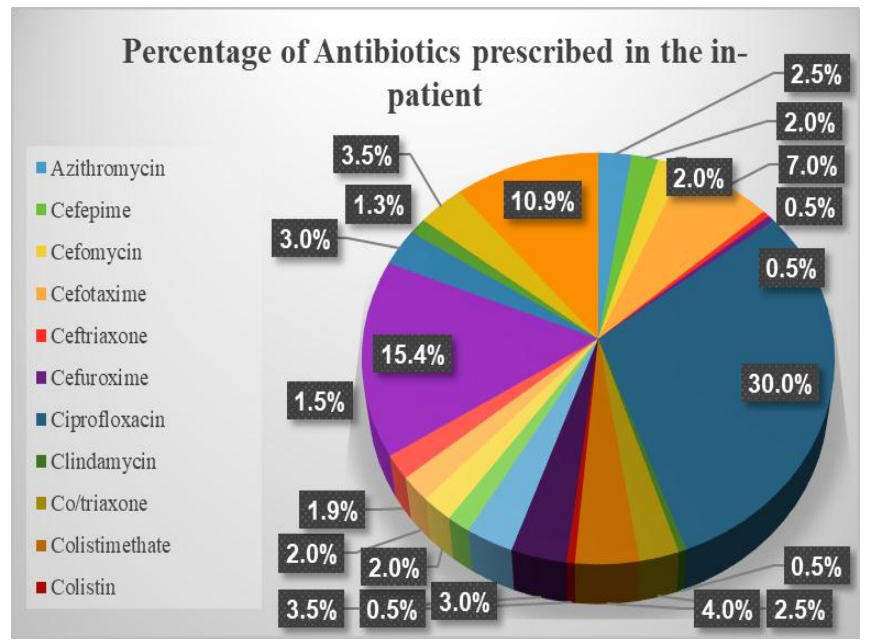

Figure 1A: Prescribed antibiotic percentages for inpatients at Hera General Hospital, (n=201 prescriptions).

A total of 7 different antibiotics alone, or in different combinations were prescribed, totalling 347 occurrences in ER. These comprised amoxicillin, augmentin, azithromycin, cefuroxime, cephalexin, ciprofloxacin and erythromycin was the most commonly prescribed antibiotic per patient either as a single agent or in combination with other antibiotics. Amoxicillin was the highest prescribed antibiotic (41\%) (Fig 1B)

\section{Percentage of Antibitoics prescribed in the ER}

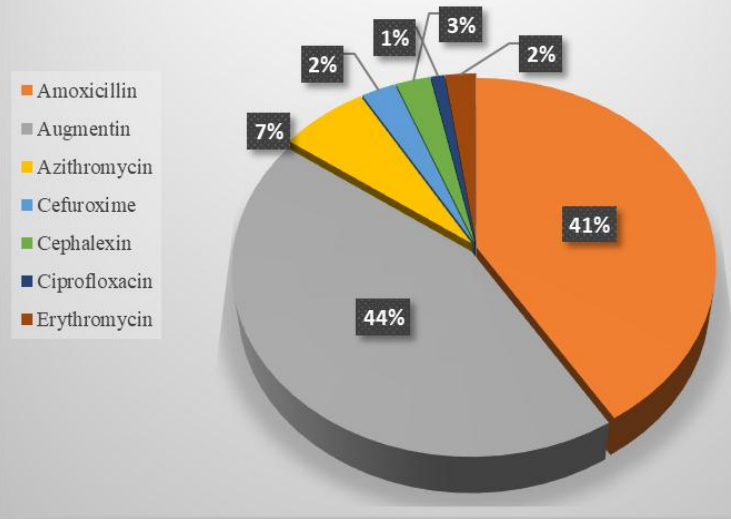

Figure 1B: Prescribed antibiotic percentages for ER at Hera General Hospital, (n=347 prescriptions).

\section{Laboratory investigations:}

Of 548 therapeutic prescriptions, $180(32.8 \%)$ were based on laboratory investigation results. Of these, 126 were done before prescriptions were given. Empirical prescriptions were based on the most likely infecting pathogen.

\section{Discussion}

Antibiotics are used for the treatment of various diseases, typically bacterial infections ${ }^{[29,30]}$. However, in previous decades, thanks to the uncontrolled misuse of antibiotics, the world has witnessed a huge evolution in the spread of microbial resistance. This impacts on society through health, economic burden, prolonged hospital stays and higher mortality rates ${ }^{[31,32]}$. Our present study showed some of the evedicne that there are elevated levels of antibiotic use, without proper laboratory investigations.
Studies from Saudi Arabia have uncovered a poor knowledge base and incorrect practice patterns among Saudi subjects. In Riyadh, approximately $77.6 \%$ of adult subjects would buy antibiotics without prescriptions ${ }^{[24]}$. Also, a high incidence of antibiotic misuse was found among $38.7 \%$ of paediatric and $57.8 \%$ of the adult emergency department at the King Abdullah international medical research centre ${ }^{[33]}$. In addition, antibiotic misuse was associated with high rates of infection in a community hospital in Saudi Arabia ${ }^{[13]}$. Reports from other Arab countries have shown high rates of antibiotic overuse, misuse and selfmedication, e.g. in Egypt, Iraq, Palestine, and Jordan ${ }^{[34,35]}$.

Since the immunity of older patients is generally poorer than that of younger adult patients, antibiotic usage might well be more frequent in older patients ${ }^{[20]}$. This was clearly shown in our study from inpatients as $60 \%$ of prescriptions were for 65 years old and above. However in contrast to the ER in the present study, we found that patients from infancy to 13 years old received more prescriptions for antibiotics than patients older than 60years.

Also, in some cases children over 12 years were regarded as adults. This was inappropriate because many 12 year olds (or older) have not been through puberty and have not reached adult height and weight ${ }^{[20]}$. This was reflected in the prescription of some antibiotics, particularly ciprofloxacin for UTIs. Children under 18 had greater chances of pain or swelling in bones, joints, or tendons (musculoskeletal) while taking ciprofloxacin. Ciprofloxacin should not be the antibiotic of first choice for children under 18 years of age. Ciprofloxacin should not be used in this group, except to treat specific serious infections such as complicated UTIs, and to prevent anthrax after exposure to the bacteria (inhalational exposure) ${ }^{[20]}$. In our study however, age and type of antibiotic was not included in the results.

Moreover, from this study, the most common UTIs were tonsillitis in the ER and bronchopneumonia in the inpatient ward. Generally, the respiratory tract comprises the upper and lower parts: the upper respiratory tract comprises the sinuses, middle ear, pharynx, epiglottis and larynx, while the lower respiratory tract consists of the structures below the larynx - the bronchi, bronchioles and alveoli ${ }^{[20,36]}$.

It is well known that the aetiology of the common cold is viral based $^{[20,36]}$. For most patients with a common cold, antibiotics are unnecessary, especially in adults. Common cold symptoms resolve without antibiotic treatment within a week. If a cold lasts longer than this and is accompanied by persistent fever and a cough with increased phlegm or offensive nasal discharge, there is a possibility of secondary bacterial infection of the respiratory tract or influenza. It is estimated that less than $10 \%$ of patients with a common cold suffer with a bacterial infection ${ }^{[2]}$. Many cases of sore throat (pharyngitis) are not due to infection but are caused by other factors, such as smoking. Where infection is the cause, most cases are of a viral nature and reflect part of the cold and flu spectrum $^{[20,36]}$.

There is only one common bacterial cause of sore throat; Streptococcus pyogenes, the group A beta haemolytic streptococcus. The aim of any diagnostic procedure is to distinguish a streptococcal sore throat, which is amenable to antibiotic treatment, from viral infections which are not. Therefore if a streptococcal infection is suspected, a throat swab should be taken for culture ${ }^{[20,36]}$. Treatment of a viral sore throat is normally 
directed at symptomatic relief, e.g. rest, antipyretics and aspirin gargles ${ }^{[20,36]}$. If streptococcal infection is suspected, treatment may be started before throat swab cultures are collected. Informal interviews have shown that antibiotic treatment of common colds and sore throats was for most situations empirical, and not based on laboratory results.

Treatments were targeted mainly at streptococcal infection. Although many busy clinicians commence treatment on clinical grounds alone, the problem of resistance has led to increasing pressure on prescribers to restrict empirical antibiotic use, particularly for conditions that are primarily viral, e.g. pharyngitis ${ }^{[20,36]}$. In our study, the commonly prescribed antibiotics were the penicillin family, especially amoxicillin + clavulanic acid and amoxycillin. From observations, prescriptions for azithromycin and cefuroxime are also increasing. There is now convincing evidence that cephalosporins are more effective in terms of clinical response and eradication of microorganisms from the oropharyn $x^{[20,36]}$.

Occasionally in children, the common cold is complicated by otitis media ${ }^{[30]}$. The diagnosis of otitis media is clinically derived, laboratory investigations have little role to pla. Treatment has to be given empirically and should be effective against the three main bacterial pathogens, Strep. Pneumoniae, Haemophilus influenzae, and S. pyogenes. The broad spectrum agent amoxicillin, which is recommended for oral treatment since it is well absorbed, was commonly prescribed in our study, in some cases for up to 14 days, even though a 10 day regiment treatment is ideal. It was prescribed for three times a day. Some prescribers preferred the more expensive amoxycillin + clavulanic acid.

For lower RTIs, e.g. non-severe pneumonia, the revised Standard Treatment Guidelines recommends, as first line treatment, $1 \mathrm{~g}$ oral amoxycillin, at 8 hourly intervals for 7 days. If the patient is allergic to penicillin, $500 \mathrm{mg}$ erythromycin at 6 hourly intervals, or $500 \mathrm{mg}$ azithromycin daily, for 7 days ${ }^{[30]}$. Virtually all prescribers in this study preferred the more expensive second line treatment with $1 \mathrm{~g}$ oral amoxicillin + clavulanic acid at 12-hourly intervalsfor 7 days. In some cases, prescribers suggested a frequency of 8 hours, which was not necessary. Diagnosis was based on chest $\mathrm{X}$-rays and full blood counts ${ }^{[36]}$.

These results suggest antibiotics are an overused medicinal commodity. This reflects a dangerous phenomenon; Davey et al. reported that high percentages of UK patients with upper respiratory symptoms were prescribed antibiotics ${ }^{[20]}$. The pattern of infections encountered was similar to that in many countries as in Saudi Arabia.

\section{Conclusions}

To reduce unnecessary antibiotic usage, it is vital to take action now to improve prescribing practices, thus enhancing a rational approach to antibiotic use. The results of this study revealed there is a need for an antibiotic formulary or clinical guidelines. A Clinic's Drugs and Therapeutics committee can play an important role in this regard; they can perform drug utilisation studies and prescription reviews to improve drug management and general use. Doctors tend to use newer, more expensive medicinal agents, as opposed to cost effective, proven, and well-established antimicrobial agents.

\section{Recommendations}

Educational programmes aimed at graduating general practitioners and newly qualified doctors should focus on the appropriate use of antibiotics. Also, legal restrictions should be enhanced for accessing antibiotics.

More studies are required to determine trends and patterns in antibiotic usage. These should identify factors influencing medical and clinical opinion, such as commercial information as provided by pharmaceutical companies and their sales representatives. Periodic surveys of antibiotic usage at the clinic should be carried out as part of nosocomial infection surveillance and control programmes. Prospective studies are preferred; more control is exerted over data collection, outcomes can be monitored in real time leading to more valid answers and conclusions.

\section{Limitations}

\section{Limitations of this study include:}

1) Not all prescription were clear with the precise diagnosis.

2) Some errors were detected in entries at the pharmacy, especially quantities of issued medicines and some double entries.

\section{References}

[1] Sneha A, MathurAK (2006). CHAPTER-2 RATIONAL DRUG USE. Health administrator 19(1): 5-7.

[2] WHO: How to develop and implement a national drug policy. - 2nd ed.Updates and replaces: Guidelines for developing national drug policies (1988). [Last accessed Jan 2018].

[3] Kunin, C.M.1993. Resistance to Antimicrobial Drugs - a Worldwide Calamity. Annals of Internal Medicine, 118(7): 557-561.

[4] Ibeawuchi, R., Mbata, T. 2002. Rational and Irrational Use of Antibiotics. Africa Health, January; 24(2): 16-8.

[5] Teng, C. B., Ng, T. M., Tan, M. W., Tan, S. H., Tay, M., Lim, S. F., \& Lye, D. C. (2015). Safety and effectiveness of improving carbapenem use via prospective review and feedback in a multidisciplinary antimicrobial stewardship programme. Ann Acad Med Singapore, 44(1), 19-25.

[6] Austin, D.J., Kristinsson, K.G., Anderson, R.M.1999. The Relationship between the Volume of Antimicrobial Consumption in the Human Communities and the Frequency of Resistance. Proc Natl Acad Sci USA, 96:1152-1156.

[7] Monnet, D.L., Harbarth, S. 2004. Antibiotic Selection Pressure and Resistance in Streptococcus Pneumonia and Streptococcus Pyogenes. Emerg Infect Dis, 10:514-517

[8] Mainous, A.G., Saxena, S., Hueston, W.J. et al. 2006. Ambulatory Antibiotic Prescribing for Acute Bronchitis and Cough and Hospital Admissions for Respiratory Infections. Journal of the Royal Society of Medicine, 99:358-362.

[9] FDA Consumer Health Information. 2008. Combating Antibiotic Resistance (article available at http://www.fda.gov/consumer/updates/antibiotics050108. html (Last accessed 18 Jan 2018).

[10] Norrby, S., Nord, C., Finch, R. 2005. Lack of Development of New Antimicrobial Drugs: a Potential 
Serious Threat to Public Health. The Lancet Infectious Diseases, 5(2):115-119.

[11] Grimaldi-Bensouda, L., Bégaud, B., Rossignol, M., Avouac, B. (2014). Management of Upper Respiratory Tract Infections by Different Medical Practices, Including Homeopathy, and Consumption of Antibiotics in Primary Care. PLoS ONE, 9(3), p.e 89990.

[12] Dasaraju, P. and Liu, C. (2018). Infections of the Respiratory System. [PubMed][Accessed 16 Mar. 2018].

[13] Alghamdi AA, Alamoudi OS, Ghabrah TM. Pattern of infectious diseases in the western region of Saudi Arabia; a study of 495 hospitalized patients. J King Abdulaziz Univ Med Sci 2009; 16:3-15.

[14] Centre for Clinical Practice at NICE (UK). Respiratory Tract Infections - Antibiotic Prescribing: Prescribing of Antibiotics for Self-Limiting Respiratory Tract Infections in Adults and Children in Primary Care. London: National Institute for Health and Clinical Excellence (UK); 2008.

[15] Gao, L., Lyu, Y., \& Li, Y. (2017). Trends in drug resistance of Acinetobacter baumannii over a 10 -year period: nationwide data from the China surveillance of antimicrobial resistance program. Chinese medical journal, 130(6), 659.

[16] Altabbal, N.Muhammad, rational use of antibiotics in Tabuk city and evaluation of the public awareness, KSA, 2017.

[17] Bosch, A., Biesbroek, G., Trzcinski, K., Sanders, E. and Bogaert, D. (2013). Viral and Bacterial Interactions in the Upper Respiratory Tract. PLoS Pathogens, 9(1), p. e1003057.

[18] Jain N, Lodha R and Kabra SK. 2001. Upper respiratory tract infections. Indian J Pediatr 68:1135-1138.

[19] Teng C, Leong K, Aljunid S, Cheah M. Antibiotic prescription in upper respiratory tract infections. Asia Pacific Family Medicine 2004;3(1-2):3845.

[20] Chang S, Chang H, Lai M: Antibiotic usage in primary care units in Taiwan; International Journal of Antimicrobial Agents 11 (1999) 23-30.

[21] Hall CB. Long CE, Schnabel KC. Respiratory syncytial virus infections in previously healthy working adults. Clinical Infectious Diseases 2001; 33:792-6.

[22] Cloete, T. (2003). Resistance mechanisms of bacteria to antimicrobial compounds. International Biodeterioration \& Biodegradation, 51(4), pp.277-282.

[23] Akkerman, A., Kuyvenhoven, M., van der Wouden, J. and Verheij, T. (2005). Determinants of antibiotic overprescribing in respiratory tract infections in general practice. Journal of Antimicrobial Chemotherapy, 56(5), pp.930-936.

[24] Bin Abdulhak, A., Al Tannir, M., Almansor, M., Almohaya, M., Onazi, A., Marei, M., Aldossary, O.,
Obeidat, S., Obeidat, M., Riaz, M. and Tleyjeh, I. (2011). Non-prescribed sale of antibiotics in Riyadh, Saudi Arabia: A Cross Sectional Study. BMC Public Health, 11(1).

[25] AlKhamees, O., AlNemer, K., Bin Maneea, M., AlSugair, F., AlEnizi, B. and Alharf, A. (2018). Top 10 most used drugs in the Kingdom of Saudi Arabia 2010 2015. Saudi Pharmaceutical Journal, 26(2), pp.211-216.

[26] Al-Khaldi Y, Diab M, Al-Gelban K, Al-Asmari A, AlAmin S, Al- Shahrani M. Prescribing Patterns for Acute Respiratory Infections In Primary Health Care, Aseer Region, Saudi Arabia. Journal of Family and Community Medicine. 2005;12(3):121-126.

[27] Salam M, Al Anazi M, Al-Jeraisy M. Prevalence and predictors of antibiotic prescription errors in an emergency department, Central Saudi Arabia. Drug, Healthcare and Patient Safety. 2015;103

[28] Hajjar, W., khelb, S., Mutairi, S. and Refayi., N. (2017). Antibiotics use and misuse in upper respiratory tract infection patients: Knowledge, attitude and practice analysis in University Hospital, Saudi Arabia. International Journal of Advanced Research, 5(2), pp.1604-1611.

[29] Lieberman JM (2003): Appropriate antibiotic use and why it is important: the challenges of bacterial resistance. The Pediatric infectious disease journal, 22: 1143-1151

[30] Aminov RI (2010): A Brief History of the Antibiotic Era: Lessons Learned and Challenges for the Future. Frontiers in microbiology, 1: 134.

[31] Penchovsky R, Traykovska M (2015): Designing drugs that overcome antibacterial resistance: where do we stand and what should we do? Expert Opin Drug Discov., 10: 631-650.

[32] Ventola CL (2015): The Antibiotic Resistance Crisis: Part 1: Causes and Threats. Pharmacy and Therapeutics, 40: 277-283.

[33] Alanazi MQ, Al-Jeraisy MI, Salam M (2015): Prevalence and predictors of antibiotic prescription errors in an emergency department, Central Saudi Arabia. Drug, healthcare and patient safety, 7: 103-111.

[34] Dooling KL, Kandeel A, Hicks LA, El-Shoubary W, Fawzi K, Kandeel Y et al. (2014): Understanding Antibiotic Use in Minya District, Egypt: Physician and Pharmacist Prescribing and the Factors Influencing Their Practices. Antibiotics (Basel, Switzerland), 3: 233-243.

[35] Jassim AM (2010): In-home Drug Storage and Selfmedication with Antimicrobial Drugs in Basrah, Iraq. Oman Med J., 25: 79-87.

[36] Chang SC, Shiu MN, Chen TJ. Antibiotic usage in primary care units in Taiwan after the institution of national health insurance. Diagn Microbiol Infect Dis 2001;40(3):137-43. 